\title{
Quantitative Analysis of Textural Features Extracted from Sonograms of Biceps under Different Physiological States
}

\author{
Lanting Jia, MD ${ }^{a, 1}$, Jiaqi Zhao, MD ${ }^{a, 1, *}$, Qi Xu, PhD ${ }^{b}$, Qian Pan, MD ${ }^{a}$, Jianquan Zhang, MD ${ }^{a}$ \\ ${ }^{a}$ Department of Ultrasound, Changzheng Hospital, Second Military Medical University, Shanghai, China; ${ }^{b}$ Department of Computer \\ Science, Institute of Information Engineering, Shanghai Maritime University, Shanghai, China
}

Received September 19, 2019; revision received February 24, 2020; accepted March 13, 2020.

Objective: To quantitatively analyze the difference of texture features of skeletal muscle in high-frequency ultrasound images under different physiological states using the multiscale decomposition method of ultrasound echo intensity interface reflections.

Methods: High frequency ultrasound images of the biceps brachii in different physiological states were collected from 20 healthy volunteers. In offline state, eight texture parameters including mean of texture gray scale (Mean), standard variance (SDev) of gray scale, number of blobs (NOB) of texture density, irregularity (IRGL) of texture primitive shape, mean size of blobs (SOB) of texture primitive, homogeneity of distribution (HOD) of texture uniformity, directionality of texture distribution (DOD), and periodicity of texture distribution (POD) were extracted by MATLAB software and compared and analyzed statistically.

Results: With the use of high frequency ultrasound, all healthy volunteers' biceps brachii showed isoechoic muscle bundles, organized arrangement of muscle fibers, and distinct and intact texture of structure. In different physiological states of biceps brachii of the same gender group, the NOB and the Mean showed statistically differences $(P<0.05)$. In the relaxation state of biceps brachii between different gender groups, the average SOB and the DOD showed statistically differences $(P<$ $0.05)$. In the load state of biceps brachii between different genders, the NOB and the Mean showed statistically differences $(P<0.05)$.

Conclusions: The ultrasonic image changes of muscle fibers under different physiological states can be identified by quantitative texture characteristic parameters, providing more information for clinical computer-aided diagnosis of skeletal muscle injury.

Key words: Muscle injury; Ultrasonography; Computer-aided diagnosis; Biceps Texture; Quantitative analysis;

Advanced Ultrasound in Diagnosis and Therapy 2020;04:183-188

DOI: 10.37015/AUDT.2020.190024

ompared with magnetic resonance imaging (MRI), high-frequency ultrasound (HFUS) has become the first choice for skeletal muscle examination because of its high resolution, lower cost and convenience [1]. Skeletal muscle is a form of striated muscle, which muscle fibers are organized into individual bundles, and hypoechoic stripe muscle bundles and hyperechoic muscle fibers are arranged in parallel position, forming unique texture features on ultrasound images. However, ultrasound scans requires experienced clinicians to get an accurate result, and the quality of the image is affected by the ultrasound performing methods and the equipment. Different ultrasound providers have subjectively differences in identifying and interpreting the manifestations of skeletal muscle ultrasound textures, and there is no quantitative index for diagnostic reference

\footnotetext{
${ }^{1}$ Co-first Author

"Correspondence Author: Department of Ultrasound, Changzheng Hospital, Second Military Medical University, 415 Fengyang Road, Shanghai, 200003, China.

e-mail: qiqiblue67@163.com
} unrestricted use, distribution and reproduction in any medium provided that the original work is properly attributed. 
[2]. Previous in vitro experiments have confirmed that the ultrasound texture analysis technology has quantitative diagnostic capability in porcine striated muscle texture recognition [3]. Our study aims to analyze the changes of high-frequency ultrasonic imaging characteristics of the biceps brachii in different physiological states and to explore the applicability of computer-assisted quantitative identification in describing human muscle textures, so as to provide more quantitative and credible basis for clinical auxiliary diagnosis of skeletal muscle injury.

\section{Materials and Methods}

\section{Subjects}

Twenty healthy volunteers who underwent biceps brachii ultrasound examination in our hospital from January 2018 to December 2018 were selected for our study, including 10 male and 10 female patients between the ages of 24 and 40-years-old. None of them had history of upper limb trauma or other congenital or metabolic muscle diseases, and all the subjects were right handedness. Based on gender, we divided our patients into male and female groups, and the average age, average body mass index (BMI), and average maximum muscle thickness (level of the biceps muscle abdomen) of each group were compared. There was no statistically significance of any comparable groups $(P>$ 0.05 ) as shown in Table 1. All participants in the study were demonstrated by experts from the Hospital Ethics Committee, and all the volunteers signed informed consent forms.

Table 1 General baseline data of subjects

\begin{tabular}{lcccc}
\hline \multirow{2}{*}{ Groups } & Age $(\mathrm{y} / \mathrm{o})$ & $\mathrm{BMI}\left(\mathrm{kg} / \mathrm{m}^{2}\right)$ & \multicolumn{2}{c}{ Thickness of muscle $(\mathrm{mm})$} \\
\cline { 4 - 5 } & & & Left & Right \\
\hline Male & $35.1 \pm 6.8$ & $23.1 \pm 0.9$ & $28.6 \pm 0.9$ & $29.1 \pm 0.5$ \\
\hline Female & $32.7 \pm 5.3$ & $22.3 \pm 1.4$ & $28.3 \pm 0.8$ & $28.9 \pm 0.5$ \\
$t$ value & -0.907 & -1.575 & -0.912 & -0.701 \\
$P$ value & 0.420 & 0.314 & 0.510 & 0.684 \\
\hline
\end{tabular}

BMI, body mass index; $y / o$, year old

\section{Ultrasound equipment}

During the whole scanning process, we adopted highfrequency ultrasound diagnostic machine (iU22, Philips, Netherlands), equipped with L12 $\sim 5$ probe $(5 \sim 12$ $\mathrm{MHz}$ ). The probe was vertically attached to the muscle surface, keeping a moderate contact without pressure, and the placement angle was not inclined. The system parameters of the machine were consistent as follows,
$70 \mathrm{db}$ grayscale gain, $55 \%$ compression, 0 compensation of the time gain, $1.0-2.5 \mathrm{~cm}$ of the focus depth, 0.1 thermal index (TI), and 0.6 mechanical index (MI).

\section{Methods}

Healthy volunteers were placed in supine position, keeping the right upper limb horizontal on the right side of the body. Under this circumstance, the biceps brachii muscles were in a state of relaxation. And then the examiner flexed forearm at right angles to upper arm to make biceps brachii in a state of tension and contraction (under static load).

In both states, we firstly performed biceps crosssectional scanning continuously, found the biceps muscle abdomen, and measured the maximum thickness of the biceps, then rotated the probe $90^{\circ}$ to scan and collect high-frequency ultrasound images of the longitudinal section of the biceps brachii muscle. In offline state, we selected region of interest (ROI) to view muscle textures clearly. All ROI had the same depth, size, and shape. Through MATLAB 7.0 software (MathWorks Co., American), each ROI was sampled three times at $60 \times$ 60 pixels with a total of 30 samples were gathered. Then each final decomposed binary image was obtained by a multiscale decomposition method of ultrasound echo intensity interface reflections [4] (Fig. 1). Eight texture feature parameters were automatically calculated for each group, including the mean of texture gray scale (Mean), standard variance (SDev) of gray scale, number of blobs (NOB) of texture density, irregularity (IRGL) of texture primitive shape, mean size of blobs (SOB) of texture primitive, homogeneity of distribution (HOD) of texture uniformity, directionality of texture distribution (DOD) and periodicity of texture distribution (POD).

\section{Statistical analysis}

All dates were analyzed by SPSS 17.0 software and tested using an independent sample $t$ test. The parameters were extracted from ROI of each group and expressed as mean \pm standard deviation (SD). Differences were defined as statistically significant when $P<0.05$.

\section{Results}

HFUS images of 20 healthy volunteers' right biceps brachii showed consistent results: all the isoechoic muscle fiber bundles showed good continuity, distinct layers, and clear texture (Fig. 2, Fig. 3). The biceps brachii texture characteristics of healthy volunteers were compared in groups according to different genders and different states of right upper limb. The comparison results of the eight texture feature parameters of the biceps brachii in the contraction and relaxation states of 
healthy volunteers in each gender group were as follows:

Compared to relaxation state, biceps brachii in contracted state showed different features on sonograms: the ROI of the male group showed statistical differences in NOB, DOD and Mean (NOB: $1.93 \pm 0.53$ vs $3.05 \pm 0.8, P=0.002$; DOD: $1.09 \pm 0.46$ vs $2.13 \pm 1.1, P=0.013$; Mean: $0.09 \pm 0.05$ vs $0.18 \pm$
$0.07, P=0.004$ ) (Table 2); The ROI of the female group showed statistical differences in NOB, POD, Mean, and SDev (NOB: $1.86 \pm 0.42$ vs $4.15 \pm 0.91, P<0.001$ POD: $0.61 \pm 0.25$ vs $1.07 \pm 0.42, P=0.009$; Mean: $0.09 \pm 0.08$ vs $0.34 \pm 0.13, P=0.000 ;$ Sdev: $0.18 \pm 0.11$ vs $0.42 \pm 0.21, P=0.005$ ) (Table 3 ).

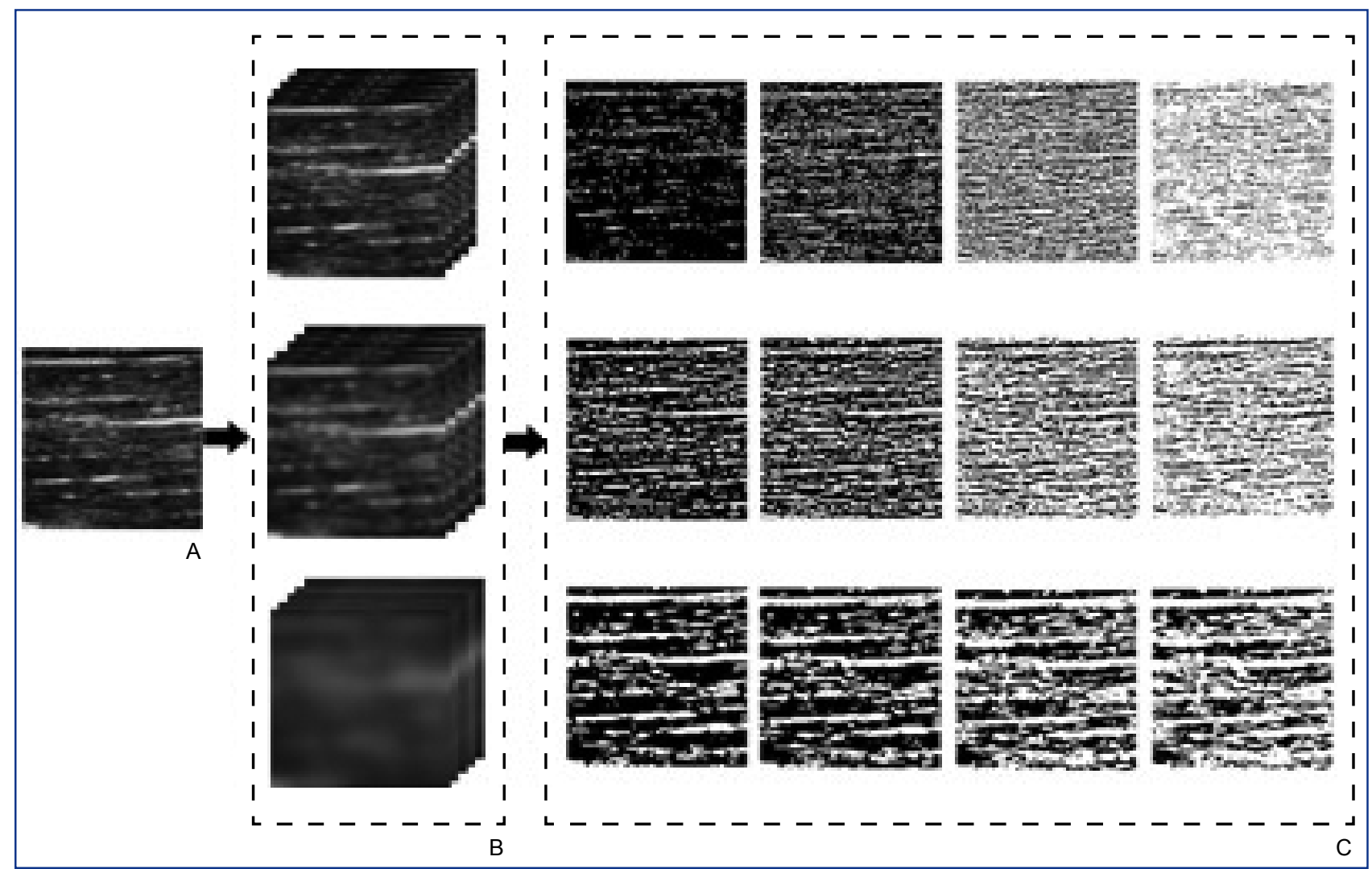

Figure 1 Schematic diagram of multistage decomposition process on skeletal muscle high frequency sonographic intensity interface. (A) Gray-scale sonogram of skeletal muscle; (B) Decomposition threshold images; (C) Intensity interface decomposed binary images

Table 2 Eight texture features of biceps brachii in male healthy volunteers under relaxation and contraction $(\bar{x} \pm s, n=30$ samples $)$

\begin{tabular}{lcccccccc}
\hline \multicolumn{1}{c}{ Parameters } & NOB & HOD & IRGL & SOB & DOD & POD & Mean & SDev \\
\hline Relaxed state & $1.93 \pm 0.53$ & $5.38 \pm 1.54$ & $5.4 \pm 0.73$ & $4.62 \pm 1.68$ & $1.09 \pm 0.46$ & $0.65 \pm 0.25$ & $0.09 \pm 0.05$ & $0.30 \pm 0.21$ \\
Contracted state & $3.05 \pm 0.8$ & $4.39 \pm 2.11$ & $5.73 \pm 1.45$ & $3.76 \pm 1.54$ & $2.13 \pm 1.1$ & $0.94 \pm 0.39$ & $0.18 \pm 0.07$ & $0.33 \pm 0.11$ \\
$t$ value & -3.715 & -1.207 & -0.641 & -1.189 & -2.751 & -1.983 & -3.254 & -0.377 \\
$P$ value & 0.002 & 0.243 & 0.529 & 0.250 & 0.013 & 0.063 & 0.004 & 0.711 \\
\hline
\end{tabular}

NOB, number of blobs of texture density; HOD, homogeneity of distribution of texture uniformity; IRGL, irregularity of texture primitive shape; SOB, mean size of blobs of texture primitive; DOD, directionality of texture distribution; POD, periodicity of texture distribution; Mean, Mean of texture gray scale; SDev, standard variance of gray scale.

Comparisons between male and female groups: when the biceps brachii was in the state of relaxation, the muscle texture elements of different genders showed statistical differences in SOB and DOD (SOB: $4.62 \pm 1.68$ vs $2.73 \pm 1.04, P=0.009$; DOD: $1.09 \pm 0.46$ vs $1.91 \pm$ $0.80, P=0.013$ ) (Table 4); when the biceps brachii was in the state of contraction (under static load), the muscle texture elements of different genders showed statistical differences in NOB and Mean (NOB: $3.05 \pm 0.8$ vs 4.15 $\pm 0.91, P=0.010$; Mean: $0.18 \pm 0.07$ vs $0.34 \pm 0.13, P=$ 0.002) (Table 5). 
Table 3 Eight texture features of biceps brachii in female healthy volunteers under relaxation and contraction $(\bar{x} \pm s, n=30$ samples $)$

\begin{tabular}{|c|c|c|c|c|c|c|c|c|}
\hline Parameters & NOB & HOD & IRGL & SOB & DOD & POD & Mean & SDev \\
\hline Relaxed state & $1.86 \pm 0.42$ & $5.19 \pm 1.51$ & $5.25 \pm 1.54$ & $2.73 \pm 1.04$ & $1.91 \pm 0.80$ & $0.61 \pm 0.25$ & $0.09 \pm 0.08$ & $0.18 \pm 0.11$ \\
\hline Contracted state & $4.15 \pm 0.91$ & $5.34 \pm 1.01$ & $5.17 \pm 0.95$ & $3.78 \pm 1.47$ & $2.10 \pm 1.32$ & $1.07 \pm 0.42$ & $0.34 \pm 0.13$ & $0.42 \pm 0.21$ \\
\hline$t$ value & -7.230 & -0.251 & -0.135 & -1.832 & -0.390 & -2.926 & -5.395 & -3.243 \\
\hline$P$ value & $<0.001$ & 0.805 & 0.894 & 0.085 & 0.702 & 0.009 & 0.000 & 0.005 \\
\hline
\end{tabular}

NOB, number of blobs of texture density; HOD, homogeneity of distribution of texture uniformity; IRGL, irregularity of texture primitive shape; SOB, mean size of blobs of texture primitive; DOD, directionality of texture distribution; POD, periodicity of texture distribution; Mean, Mean of texture gray scale; SDev, standard variance of gray scale.
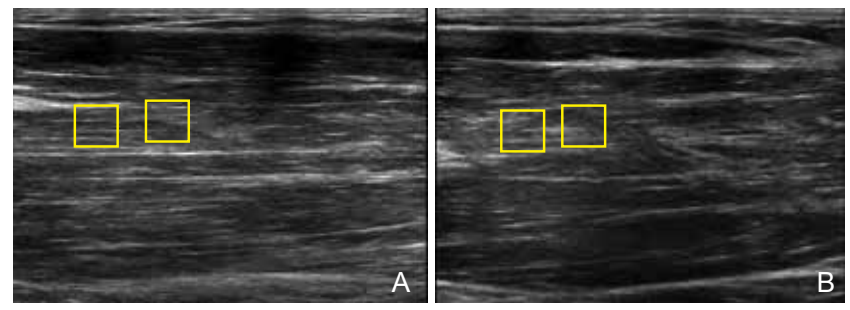

Figure 2 Textural characteristics changes of biceps brachii on high frequency ultrasonograms in healthy male volunteers under different physiological states (yellow box indicated ROI). (A) Biceps brachii relaxation without load bearing; (B) Biceps brachii contraction in load state
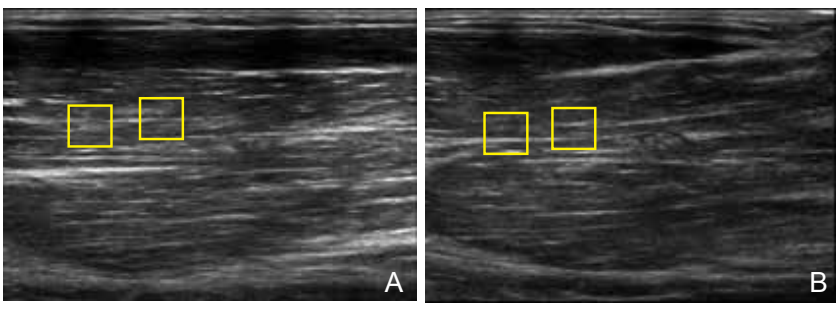

Figure 3 Textural characteristics changes of biceps brachii on high frequency ultrasonograms in healthy female volunteers under different physiological states (yellow box indicated ROI). (A) Biceps brachii relaxation without load bearing; (B) Biceps brachii contraction in load state

Table 4 Eight texture features of biceps brachii in healthy volunteers of different genders under relaxation $(\bar{x} \pm s, n=30$ samples $)$

\begin{tabular}{|c|c|c|c|c|c|c|c|c|}
\hline Parameters & NOB & HOD & IRGL & SOB & DOD & POD & Mean & SDev \\
\hline Male & $1.93 \pm 0.53$ & $5.38 \pm 1.54$ & $5.4 \pm 0.73$ & $4.62 \pm 1.68$ & $1.09 \pm 0.46$ & $0.65 \pm 0.25$ & $0.09 \pm 0.05$ & $0.30 \pm 0.21$ \\
\hline Female & $1.86 \pm 0.42$ & $5.19 \pm 1.51$ & $5.25 \pm 1.54$ & $2.73 \pm 1.04$ & $1.91 \pm 0.80$ & $0.61 \pm 0.25$ & $0.09 \pm 0.08$ & $0.18 \pm 0.11$ \\
\hline$t$ value & -0.309 & -0.278 & -0.289 & -3.016 & -2.816 & -0.361 & -0.071 & -1.644 \\
\hline$P$ value & 0.761 & 0.784 & 0.776 & 0.009 & 0.013 & 0.722 & 0.945 & 0.118 \\
\hline
\end{tabular}

NOB, number of blobs of texture density; HOD, homogeneity of distribution of texture uniformity; IRGL, irregularity of texture primitive shape; SOB, mean size of blobs of texture primitive; DOD, directionality of texture distribution; POD, periodicity of texture distribution; Mean, Mean of texture gray scale; SDev, standard variance of gray scale.

Table 5 Eight texture features of biceps brachii in healthy volunteers of different genders under contraction $(\bar{x} \pm s, n=30$ samples $)$

\begin{tabular}{|c|c|c|c|c|c|c|c|c|}
\hline Parameters & NOB & HOD & IRGL & SOB & DOD & POD & Mean & SDev \\
\hline Male & $3.05 \pm 0.80$ & $4.39 \pm 2.11$ & $5.73 \pm 1.45$ & $3.76 \pm 1.54$ & $2.13 \pm 1.1$ & $0.94 \pm 0.39$ & $0.18 \pm 0.07$ & $0.33 \pm 0.11$ \\
\hline Female & $4.15 \pm 0.91$ & $5.34 \pm 1.01$ & $5.17 \pm 0.95$ & $3.78 \pm 1.47$ & $2.10 \pm 1.32$ & $1.07 \pm 0.42$ & $0.34 \pm 0.13$ & $0.42 \pm 0.21$ \\
\hline$t$ value & -2.874 & -1.286 & -1.025 & -0.022 & -0.047 & -0.675 & -3.631 & -1.212 \\
\hline$P$ value & 0.010 & 0.221 & 0.321 & 0.982 & 0.963 & 0.508 & 0.002 & 0.246 \\
\hline
\end{tabular}

NOB, number of blobs of texture density; HOD, homogeneity of distribution of texture uniformity; IRGL, irregularity of texture primitive shape; SOB, mean size of blobs of texture primitive; DOD, directionality of texture distribution; POD, periodicity of texture distribution; Mean, Mean of texture gray scale; SDev, standard variance of gray scale.

\section{Discussion}

For a long time, MRI has been the modality of choice for evaluating muscular lesions. However, with the technology development of ultrasound, HFUS has gradually become an essential imaging method for evaluating skeletal muscle morphology, and it could further localize muscle lesions and provide accurate clinical information for muscular diseases such as muscle injuries. HFUS has comparable performance to MRI in resolving the morphological structure and the distribution of skeletal muscle bundles. Moreover, HFUS has the advantages of real-time detection, portability, 
controllability, cost-effective, and time-saving [5]. Therefore, in this study, images of HFUS were selected to conduct quantitative analysis of muscle texture under computer vision using computer image processing technology, which is expected to become an auxiliary diagnostic tool for quantitative analysis of diseases such as muscle injury, etc.

Physical training (fitness exercise), occupational work, traffic accidents, or other exogenous blunt injuries can cause muscle injured. Some scholars have used HFUS to identify the degree of upper limb muscle injury which was clinically suspected to be a contusion [6]. Zhou et al. utilized HFUS to diagnosis of muscle herniation and was confirmed by surgery. They confirmed that HFUS can be used to observe the shape, size, location, internal echoes and fascial defect of the mass of muscle herniation in a dynamic way [7].

The diagnosis of muscular lesions by HFUS should combine both qualitative and quantitative indicators. At present, many scholars at home and abroad have reached a consensus on the qualitative research of musculoskeletal ultrasound imaging. Qi et al. [8] conducted a retrospective study on the ultrasound image characteristics of 21 patients with rhabdomyolysis, and images showed that the muscular texture in the lesion area was "foggy" or "frosted glass-like" blurred with echo enhancement and irregular shape. Color Doppler flow imaging (CDFI) showed no blood flow (grade 0) or only a small amount of star-like blood flow (grade I) in the lesion area. Fornage et al. pointed out that blurring or even disappearance of muscle texture was a typical manifestation of rhabdomyolysis ultrasound images [9]. Liu et al. [10] used multi-section and multi-angle HFUS to comprehensively and dynamically scan the changes of tissue repair after acute muscle injury in 52 patients. Through the comparison with normal muscle, the study found a significant difference in echo changes of injured muscle on day 0 , day 1, 3 days after injury, which presented with interrupted continuity of muscle texture and hyperechoic muscular tissue. On day 7 and day 14 after the injury, the injured muscle showed nearly isoechoic with the adjacent muscle.

Although HFUS could describe the image features of muscle tissue injury with relatively accurate location and characteristics, it is difficult to concisely quantify the overall trend of muscle echo intensity and texture changes in a certain period of time. Therefore, our study conducted preliminarily explored the combination of quantitative and qualitative parameters in order to provide comprehensive and objective ultrasound image information for muscle injury [11].

In addition, both the relaxation and contraction states should also be taken into consideration when analyzing muscle injuries because the characteristics of muscle fibers under load are different from relaxation state $[12,13]$. In our study, biceps brachii was the main motor of upper limb skeletal muscle movement. Compared to the relaxation state, when biceps brachii was under a certain static load due to tension contraction, the ROI of male volunteers showed a statistically significance in NOB, DOD, and Mean (all $P<0.05$ ), while in female group, the NOB, DOD, POD, and SDev had statistical differences (all $P<0.01$ ). In both male and female groups, the NOB and Mean of texture changed significantly during muscular contraction.

Naked eyes couldn't distinguish the fine differences of muscle density and strength between different groups of ultrasonic images. However, it is easier to identify them by means of computer-aided quantitative detection. Specifically, the extraction of mathematical geometric features of texture under computer vision could improve the diagnostic accuracy of ultrasound. In our study, healthy volunteers' muscle textures in different physiological condition were quantitatively analyzed by computer. We found that when the biceps muscle was in the state of relaxation, muscle texture elements of different genders had statistical differences in SOB and DOD (all $P<0.05$ ); when the biceps muscle was in the state of contraction (under static load), the Mean and NOB had statistical differences between different genders (all $P<0.05$ ). Therefore, it could be inferred that different physiological state of muscle fiber and the changes of muscle texture on ultrasonograms of different genders could be accurately identified by quantitative indicators, and computer technology could be used to quantitatively detect the subtle differences of these ultrasonic features. This clinical, preliminary attempt will be an effective alternative and complementary in the field of ultrasound diagnosis of skeletal muscle lesions. In the future, in-depth studies on the occurrence, development, and repair and regeneration of clinical skeletal muscle injury should be further explored to provide more information.

Our study has several limitations. As we know, skeletal muscle is the largest metabolic exercise organ in human body. The morphological and structural characteristics of skeletal muscle are affected by many factors. For example, long-term regular and targeted loading can lead to local skeletal muscle hypertrophy, while aging could cause the loss of skeletal muscle after reaching the peak value. Obesity and diabetes can cause skeletal muscle remodeling and metabolic changes. However, our study only focused on young healthy people. In the future, more comprehensive analysis should be carried out considering other influencing factors such as obesity, age, etc. Also, due to the relatively small 
sample size of our study, muscle texture characteristics from various injury types were not discussed. More large-sized and high quality studies focusing on various muscle injury types using quantitative parameters based on pathophysiological mechanisms are needed.

With the integrated development of ultrasonic diagnostic equipment and artificial intelligence, computer technology has been used to assist quantitative analysis of muscle ultrasonic images to avoid deviation of subjective diagnosis by ultrasonic examiners. However, this method requires a careful combination of clinical medicine and industrial technology. Qualitative ultrasonic description is still the standard method, and it is hard to be replaced in the short term. Therefore, it is necessary to continue exploring the feasibility and applicability of quantitative diagnostic which is much more challenging but accurate. At the same time, we should enhance the training of musculoskeletal ultrasound subspecialty. With the prosperity of cloud storage and cloud computing technology, personalized deep learning, and image data sharing technique [14-17], more and more accurate ultrasonic imaging diagnostic tools will emerge, which will be expected to eliminate the subjective errors in the process of acquiring and interpreting ultrasound images [18], and it could further provide more comprehensive ultrasound assessment for musculoskeletal system.

\section{Acknowledgment}

This work was supported by grant from the National Natural Science Foundation of China (Grant No. 81501492).

\section{Conflicts of Interest}

The authors declare that they have no competing interests.

\section{References}

[1] Blasi M, De la Fuente J, Pérez-Bellmunt A, Zabalza O, Martínez S, et al. High-resolution ultrasound in the assessment of the distal biceps brachii tendinous complex. Skeletal Radiol 2019;48:395-404.

[2] Zhao JQ, Zhang JQ, Sheng JG, Zhao LL, Pan Q. Analysis of the impact of ultrasographer's experience in diagnosing porcine striped muscle injury from thermal and chemical factors. Chin J Med Ultrasound (Electronic Edition) 2014;11:63-68. DOI: 10.3877/cma. j.issn.1672-6448.2014.09.016. [In Chinese]

[3] Zhao J, Zhang J, Xu Q, Sheng J, Diao Z, Liu S. Quantitative evaluation of striated muscle injury by multiscale blob features method. J Med Ultrason (2001) 2016;43:337-45.

[4] Zhao JQ, Zhang JQ, Xu Q, Chen YQ, Sheng JG, Lu F, et al. A quantitative study on ultrasonographic texture analysis of injured porcine striped muscle induced by thermal and chemical factors Chin J Med Ultrasound (Electronic Edition) 2013;10:674-680. DOI: 10.3877/cma.j.issn.1672-6448.2013.08.017. [In Chinese].

[5] Woodhouse JB, McNally EG. Ultrasound of skeletal muscle injury: an update. Semin Ultrasound CT MR 2011;32:91-100.

[6] Drakonaki EE, Sudoł-Szopi ń ska I, Sinopidis C, Givissis P. High resolution ultrasound for imaging complications of muscle injury: Is there an additional role for elastography? J Ultrason 2019;19:137144.

[7] Zhou X, Zhan W, Chen W, Wu D, Wei K, Wu C, Wang L. The value of ultrasound in the preoperative diagnosis of muscle herniation: A comparison with magnetic resonance imaging. Eur J Radiol 2017; 94: 191-194.

[8] Qi HT, Teng JB, Zhang XD, Wang ZT, Yuan H. Value of color Doppler ultrasonography in diagnosis of rhabdomyolysis. Chin $J$ Ultrasonogr 2012, 21: 511-513. DOI: 10.3760/cma.j.issn.1004-4477. 2012.06.018. [In Chinese].

[9] Fornage BD, Nerot C. Sonographic diagnosis of rhabdomyolysis. $J$ Clin Ultrasound 1986;14:389-392.

[10] Liu T. Value of high-frequency ultrasound in evaluating the dynamic changes of tissue repair after acute muscle injury. China Medical Device Information 2017, 23: 106-107. DOI: 10.3969/ j.issn.1006-6586.2017.20.053. [In Chinese].

[11] Molinari F, Caresio C, Acharya UR, Mookiah MR, Minetto MA. Advances in quantitative muscle ultrasonography using texture analysis of ultrasound images. Ultrasound Med Biol 2015; 41: 25202532.

[12] Katakis S, Barotsis N, Kastaniotis D, Theoharatos C, Tsiganos P, Economou G, et al. Muscle Type and Gender Recognition Utilising High-Level Textural Representation in Musculoskeletal Ultrasonography. Ultrasound Med Biol 2019;45:1562-1573.

[13] Carlier PG, Marty B, Scheidegger O, Loureiro de Sousa P, Baudin PY, Snezhko E, et al. Skeletal Muscle Quantitative Nuclear Magnetic Resonance Imaging and Spectroscopy as an Outcome Measure for Clinical Trials. J Neuromuscul Dis 2016;3:1-28.

[14] Lemaître G, Martí R, Freixenet J, Vilanova JC, Walker PM, Meriaudeau F. Computer-Aided Detection and diagnosis for prostate cancer based on mono and multi-parametric MRI: a review. Comput Biol Med 2015; 60: 8-31.

[15] de Carvalho Filho AO, Silva AC, de Paiva AC, Nunes RA, Gattass M. Computer-aided diagnosis system for lung nodules based on computed tomography using shape analysis, a genetic algorithm, and SVM. Med Biol Eng Comput 2017;55:1129-1146.

[16] Moon WK, Huang YS, Lo CM, Huang CS, Bae MS, Kim WH, et al. Computer-aided diagnosis for distinguishing between triple-negative breast cancer and fibroadenomas based on ultrasound texture features. Med Phys 2015; 42: 3024-3035.

[17] Martel AL, Hosseinzadeh D, Senaras C, Zhou Y, Yazdanpanah A, Shojaii R, et al. An Image Analysis Resource for Cancer Research: PIIP-Pathology Image Informatics Platform for Visualization, Analysis, and Management. Cancer Res 2017; 77: e83-e86.

[18] Zhao JQ, Diao ZP, Xu Q, Zhang JQ. New development of ultrasound medicine in the era of artificial intelligence. Academic Journal of Second Military Medical University 2019; 40: 478-482. DOI: 10.16781/j.0258-879x.2019.05.0478. [In Chinese]. 